\title{
Algorithm of Context Inconsistency Elimination Based on Feedback Win- dowing and Evidence Theory for Smart Home
}

\author{
Hongji $\mathrm{Xu}^{1,2,3, *}$, Zhigang $\mathrm{Xie}^{1}$ and Leitao Wang ${ }^{1}$ \\ ${ }^{1}$ School of Information Science and Engineering, Shandong University, Jinan 250100, China \\ ${ }^{2}$ Department of Digital Media, Inspur Co., Ltd., Jinan 250101, China \\ ${ }^{3}$ Linking Photoelectron Information Technology, Co., Ltd., Jinan 250101, China
}

\begin{abstract}
Due to the accuracy difference of sensors, equipment heterogeneity, network delay and diversity of algorithms etc., inconsistency of context information may emerge, which would affect the accuracy and reliability of context reasoning and decision making. To solve this problem, an algorithm of context inconsistency elimination which is based on feedback windowing and evidence theory is proposed in this paper and can be applied in many scenarios, like smart home. Simulation results show that the proposed algorithm outperforms the other counterparts in accuracy when errors especially burst errors occur, and it can improve the accuracy and reliability of context-aware system.
\end{abstract}

Keywords: Context-aware, evidence theory, feedback, inconsistency elimination, windowing.

\section{INTRODUCTION}

Along with the popularization and application of some new technologies such as pervasive computing, internet of things and intelligent sensing, context-aware technology has become a hot research topic recently and has been applied in many different fields, like smart home, smart classroom, etc. [1-2]. Context-aware technology is a powerful technology to support the perceptual smart space, and in that space it can perceive environmental information and users' requirement actively, and then provide users with their needed services and enable them to control intelligent devices conveniently [3-4].

Context-aware system normally works on the premise that it obtains the correct context information [5]. However, due to environmental noise, precision difference of sensors, heterogeneity of devices, delay of network and difference of statistical algorithms etc., the context information collected by context-aware system is often inconsistent, which would cause the system making wrong reasoning and judgment, so we need to eliminate the context inconsistencies [6-7]. By eliminating the context inconsistencies, context-aware system can improve the consistency of context information, so that the validity of the subsequent context reasoning and reliability of context-aware system can be achieved.

Context inconsistency problem has attracted lots of attention. Some algorithms have been proposed to solve this problem. Zheng D. provides a voting based algorithm (MVote) [8] which selects the context information by plurality; Manzoor A. proposes a trust-worthiness based algorithm (MTrust) [9], and it outputs context information acquired by the sensor which has the largest perceptual precision among all context information; Dempster A. P. and his student Shafer G. present a new approach based on the improved evidence theory (M-DS) [10], and it outputs the context information contained in the proper subset which has the highest value of belief degree within the recognition framework; Lee B. H. proposes a user feedback-based context inconsistency elimination algorithm $(\mathrm{M}-\mathrm{Fb})[11]$, and it calculates the feedback error rate based on the feedback context data and defines accepted threshold and rejection threshold, and takes the ideas of both M-Vote and M-Trust approaches into consideration.

Nevertheless, M-Vote algorithm can not effectively suppress the influence of low accuracy context data on system; M-Trust algorithm just utilizes the context information which has the lowest error rate, and cannot make full use of all context information; the algorithm complexity of M-DS algorithm is too high, and it has no self adaptability; $\mathrm{M}-\mathrm{Fb}$ algorithm could not obtain ideal performance for some conditions. For the above four algorithms, when the context information acquired by sensors only contains random errors, they can deal with the context inconsistencies effectively. However, there are always some burst errors in contextaware information by reason of environmental interference, limitation of sensor operating range, sensor life limitation and network malfunction. When dealing with burst errors, the processing results of the above four algorithms would be not very satisfactory. 


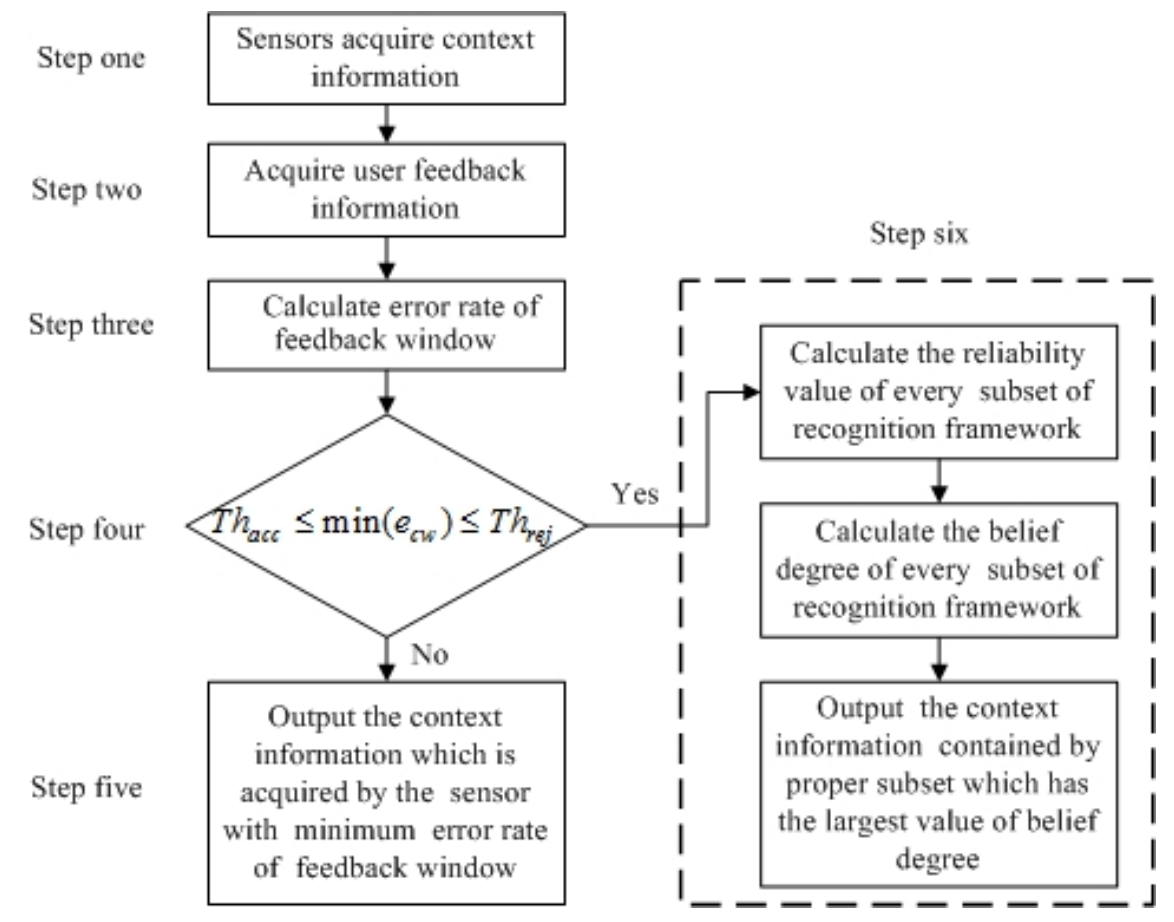

Fig. (1). The process of context inconsistency elimination algorithm based on feedback windowing and evidence theory.

\section{CONTEXT INCONSISTENCY ELIMINATION AL- GORITHM BASED ON FEEDBACK WINDOWING AND EVIDENCE THEORY}

In view of the deficiency of the existing context inconsistency elimination algorithms, this paper introduces the windowing mechanism to enhance context-aware system's ability of resisting burst errors through a rectangular window, and applies the evidence theory to further improve the accuracy of context inconsistency elimination. The proposed algorithm would be discussed as follows:

Firstly, we define several parameters here:

Definition 1: Error rate of feedback window

$e_{c w}=\frac{\text { Incon_number }}{W_{\text {_length }}}$

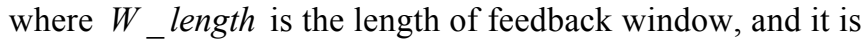
the number of the feedback data which is nearest to the statistics moment, and Incon_number is the number of inconsistent data between the original acquired context information and the feedback context information within the length of feedback window.

Definition 2: Acceptance threshold of $e_{c w}$

We define the acceptance threshold of $e_{c w}$ as $T h_{a c c}$, and suppose that context information is credible when $e_{c w}$ is lower than $T h_{a c c}$.

Definition 3: Rejection threshold of $e_{c w}$
We define the rejection threshold of $e_{c w}$ as $T h_{r e j}$, and suppose that context information is not credible when $e_{c w}$ is higher than $T h_{r e j}$.

The basic idea of the proposed context inconsistency elimination algorithm in this paper can be summarized as follows: Firstly, detecting the inconsistency of context information acquired by sensors; Secondly, calculating $e_{c w}$; Finally using $T h_{a c c}, T h_{r e j}$ and inconsistency elimination processing to remove the context inconsistencies. The process of the proposed algorithm is as follows, and the flowchart is shown in Fig. (1):

\section{Step One:}

Context-aware system acquires context information by sensors, and then executes some necessary pre-processing.

\section{Step Two:}

Context-aware system acquires feedback information.

\section{Step Three:}

According to the pre-configured length of feedback window, context-aware system counts the number of inconsistency between context information acquired by sensors and the feedback context information within the window length, and then calculates $e_{c w}$. 
Table 1. Basic reliability value of each subset of recognition framework.

\begin{tabular}{|c|c|c|c|c|c|}
\hline Subset & Subset $C_{1}$ & Subset $C_{2}$ & $\ldots$ & subset $C_{n}$ & $\begin{array}{c}\text { Complete } \\
\text { Subset } C_{c s}\end{array}$ \\
\hline \hline Basic reliability value & $m_{1}$ & $m_{2}$ & $\ldots$ & $m_{n}$ & $m_{c s}$ \\
\hline
\end{tabular}

\section{Step Four:}

We define the minimum value of $e_{c w}$ of all sensors as $\min \left(e_{c w}\right)$. Context-aware system compares $\min \left(e_{c w}\right)$ with $T h_{a c c}$ and $T h_{r e j}$. If $\min \left(e_{c w}\right)$ is lower than $T h_{a c c}$ or higher than $T h_{r e j}$, then go to step five; else go to step six.

\section{Step Five:}

Use the credibility-based context inconsistency elimination algorithm to eliminate context inconsistencies [9]. This algorithm outputs context information acquired by the sensor which has the minimum error rate of feedback window.

\section{Step Six:}

Context-aware system uses Dempster-Shafer evidence theory (DST) [12] to eliminate the context inconsistencies. In DST, there are four definitions:

\section{Definition 1: Recognition framework}

The recognition framework $\Theta$ can be seen as an incompatible and exhaustive collection of propositions in an experiment.

Definition 2: Basic reliability assignment function

For any subset $A$ which belongs to the recognition framework $\Theta$, if it meets

$$
\left\{\begin{array}{c}
m(\phi)=0 \\
\sum_{A \subset \Theta} m(A)=1
\end{array}\right.
$$

the function $m$ will be called as the basic reliability assignment function on the recognition framework $\Theta$. Meanwhile $m(A)$ is the basic reliability assignment value of subset $A$, and will be called as the focal element of the recognition framework $\Theta$ if $m(A)>0$.

\section{Definition 3: Belief function}

For any subset $X$ and $Y$ which belong to the recognition framework $\Theta$, the belief function can be defined as:

$$
\operatorname{Bel}(X)=\sum_{Y \subseteq X} m(Y)
$$

\section{$\operatorname{Bel}(X)$ reflects the belief degree of subset $X$.}

Definition 4: The basic reliability synthesis principle

We assume:
(1) $m_{1}$ and $m_{2}$ are different basic reliability assignment functions on the same recognition framework $\Theta$.

(2) The focal elements of $m_{1}$ are $A_{1}, A_{2}, \ldots, A_{k}$, meanwhile the focal elements of $m_{2}$ are $B_{1}, B_{2}, \ldots, B_{k}$.

Dempster's basic reliability synthesis principle can be expressed as

$m(C)=\frac{\sum_{A_{i} \cap B_{j}=A} m_{1}\left(A_{i}\right) m_{2}\left(B_{j}\right)}{1-K}$

Subject to: $K=\sum_{A_{i} \cap B_{j}=\phi} m_{1}\left(A_{i}\right) m_{2}\left(B_{j}\right)$

$m(C)$ reflects the joint support degree for proposition by evidence $m_{1}$ and evidence $m_{2}$. Basic reliability synthesis principle satisfies commutative law and associative law, so it can be repeatable used to calculate synthesis reliability assignment of multiple basic reliability assignment functions.

Concrete steps of evidence theory can be described as follows:

Firstly, according to the context information of each sensor, establish the recognition framework. Here we assume that the recognition framework is $\Theta=\left\{C_{1}, C_{2}, C_{3} \cdots C_{n}\right\}$.

Secondly, base on each sensor's $e_{c w}$, determine their basic reliability assignment functions of recognition framework independently.

The reliability allocation method of basic reliability assignment function is shown in Table $\mathbf{1}$ :

Basic reliability values of every subset of recognition framework satisfy the following formula:

$m_{1}+m_{2}+\cdots+m_{n}+m_{c s}=1$

For a single sensor, we calculate its $e_{c w}$ first. If current context information acquired by this sensor is $C_{i}$, basic reliability value of each subset of recognition framework can be assigned as follows:

$$
m_{j}=\left\{\begin{array}{ll}
1-e_{c w} & j=i, \\
0 & j \neq i, \\
e_{c w} & C_{j}=C_{c s}
\end{array} \text { where } C_{j} \text { is the subset of } \Theta\right.
$$

Thirdly, utilize the basic reliability synthesis principle to calculate synthesis reliability assignment of multiple basic 
reliability assignment functions. Then calculate the belief degree of every subset of recognition framework.

Finally, output the context information contained by proper subset which has the largest value of belief degree.

\section{SIMULATIONS AND ANALYSIS}

The proposed algorithm in this paper is suitable for different context-aware occasions in smart home, such as identity recognition, identification of user status, adjustment of indoor temperature, etc.

Here we assume the simulation environment of the context-aware system as follows: Five sensors are used to acquire context information to recognize user's identity, which include a RFID sensor, three cameras and a microphone. They conduct the identification through the user identity card, user images and sound respectively, and the three cameras take photos of the objective user from different angles.

Assume that the errors in the acquired context data of different sensors can be divided into two types, i.e., type 1: only random errors; type 2: random errors plus burst errors.

Suppose that when user A enters home, all sensors begin to collect his context information simultaneously, and then context-aware system is used to recognize the user's identity. We suppose that all five sensors collect 20000 user context data respectively, and for the sake of stability, the simulation would be done for 2000 times repeatedly.

As some parameters in the proposed algorithm, such as $T h_{a c c}, T h_{r e j}$, the feedback rate and the length of feedback window, would affect accuracy of the algorithm significantly, we try to obtain the optimal value of each parameter through the following several groups of related simulations. As mentioned above, when the error rate of certain sensor exceeds the rejection threshold, the context data acquired by this sensor is not credible. Here we set $T h_{r e j}$ to be $50 \%$ which is corresponding to the experience value in normal simulations, and the other parameters would be determined by the following simulations.

In practical application scenarios, there would be some burst errors due to interference or some other factors. We can evaluate the performance of context inconsistency elimination algorithm based on different kinds of burst errors. Here we assume that the system acquires six groups of data in which three groups contain burst errors while the others do not, which is very practical and common, and thus we make it as an example for analysis.

For different applications, the methods of dividing error rate interval are different, and here the error rate interval is divided into three types based on the experimental environment of smart home, i.e., a) $0-20 \%$ is the small error rate range; b) $20-30 \%$ is the middle error rate range; c) More than $30 \%$ is the large error rate range. The typical conditions of simulation data in this paper are shown in Table 2: (1) In the first and the fourth groups, the error rates of all five sensors are relatively small, and the typical values are set to be $15 \%$;
(2) In the second and the fifth groups, the error rates of five sensors are in different ranges, and the typical values are set to be $15 \%, 25 \%$ and $35 \%$, respectively; (3) In the third and the sixth groups, the error rates of all five sensors are relatively large, and the typical values are set to be $35 \%$. Here we set the length of burst errors as 300 , which conforms to some actual situations.

Through simple simulations, we can know that the optimal value of $T h_{a c c}$ is $5 \%$. Due to the space limitation, we just give the final value of it and would not introduce the intermediate simulation process, thus the main effort would focus on the most critical parameter: the length of feedback window.

In the following simulations, we will analyze the relationship between the length of feedback window and the algorithm accuracy, and get the optimal value of the length finally. Here $T h_{a c c}$ and $T h_{r e j}$ are set to be 5\% and 50\% respectively, and the feedback rate is chosen as $10 \%, 20 \%$ and $30 \%$ respectively.

From Fig. (2a) we can see that if the feedback rates correspond to $20 \%$ and $30 \%$, the optimal values of window length are 40 ; if the feedback rate corresponds to $10 \%$, the optimal value of window length is 30 . When the length of feedback window is smaller than the optimal value, the accuracy of algorithm decreases; and when the length of window is larger than the optimal value, the accuracy of algorithm also decreases; and when the length of window exceeds 600 , the accuracy of algorithm becomes stabilized.

From Fig. (2b) we can see that the accuracy of the proposed algorithm is highest when the length of window is 20 ; and when it is lower than 20, the accuracy decreases; and when the length of window is larger than 20, the accuracy also decreases; and when the length of window exceeds 600 , the accuracy becomes stabilized.

From Fig. (2c) we can see that if the feedback rate corresponds to $30 \%$, the optimal value of window length is 50 ; if the feedback rate corresponds to $20 \%$, the optimal value of window length is 30 ; if the feedback rate corresponds to 10 $\%$, the optimal value of window length is 20 . When the length of window is smaller or larger than the corresponding optimal value, the accuracy decreases; and when the length of window exceeds 600 , the accuracy becomes stabilized.

By comprehensive analysis for Fig. (2a-c), it can be seen that if the data contain both random errors and burst errors, the accuracy of the proposed algorithm is relatively higher when the length of feedback window is between 20 and 100 . When the length of window is smaller than the optimal value, the accuracy of algorithm decreases because the length of window is too short to reflect the situation of random errors of data; and when the length of window is larger than the optimal value, the accuracy also decreases because the ability of the algorithm to identify burst errors reduces; and when the length of window exceeds 600 , the accuracy becomes stabilized because the feedback error rate can truly reflect the condition of the random errors, but it is not sensitive to burst errors. 


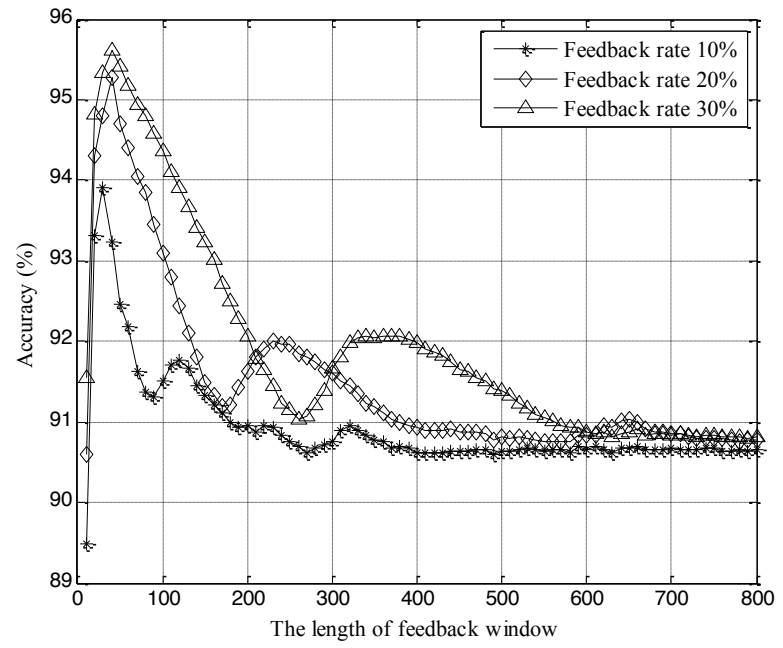

Fig. (2a). The accuracy versus the length of feedback window for the $1^{\text {st }}$ group of data.

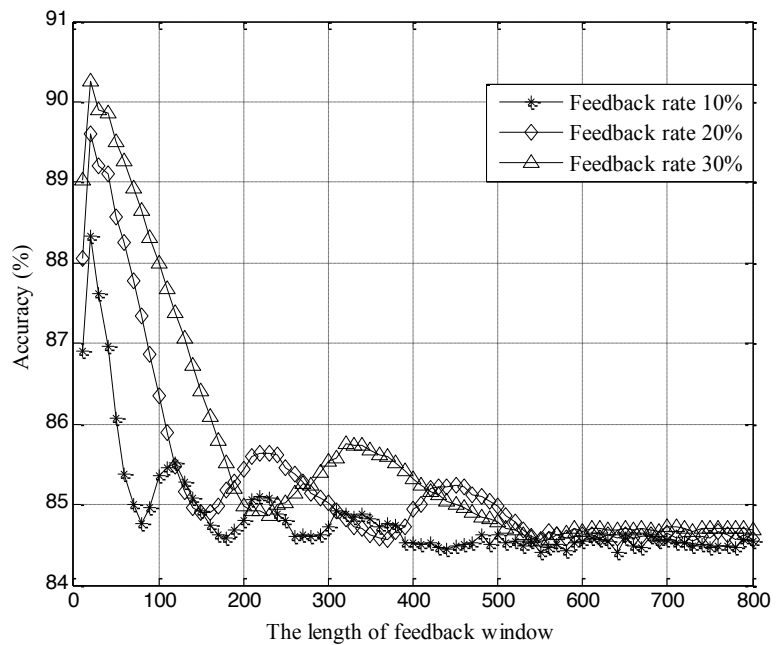

Fig. (2b). The accuracy versus the length of feedback window for the $2^{\text {nd }}$ group of data.

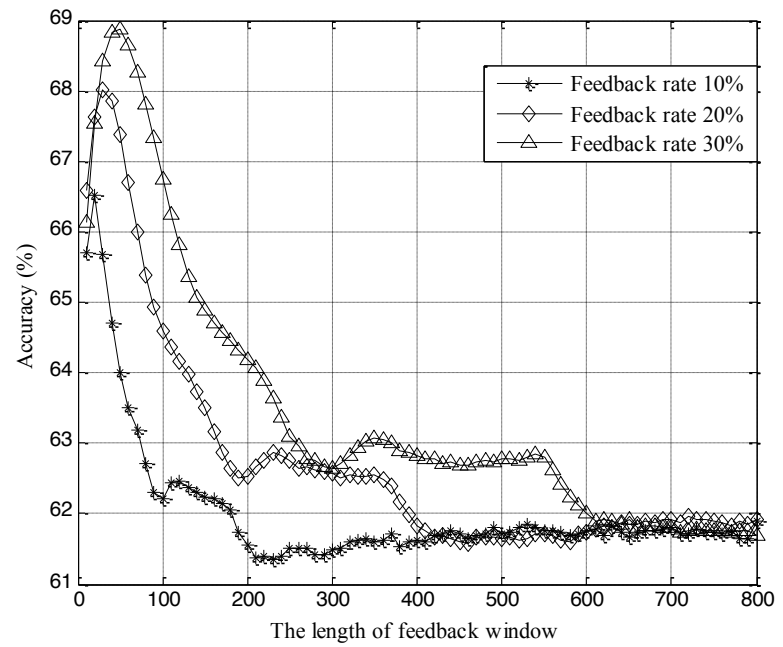

Fig. (2c). The accuracy versus the length of feedback window for the $3^{\text {rd }}$ group of data. 


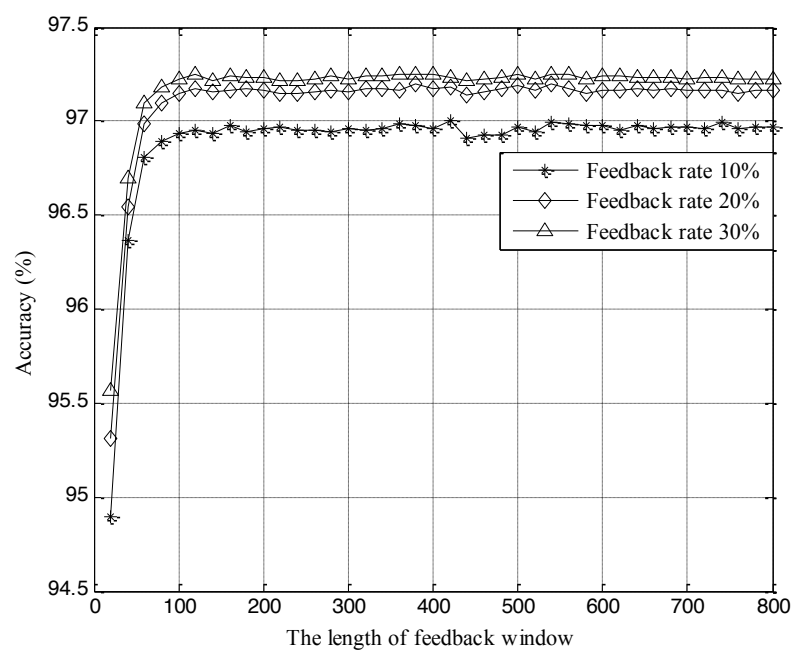

Fig. (2d). The accuracy versus the length of feedback window for the $4^{\text {th }}$ group of data.

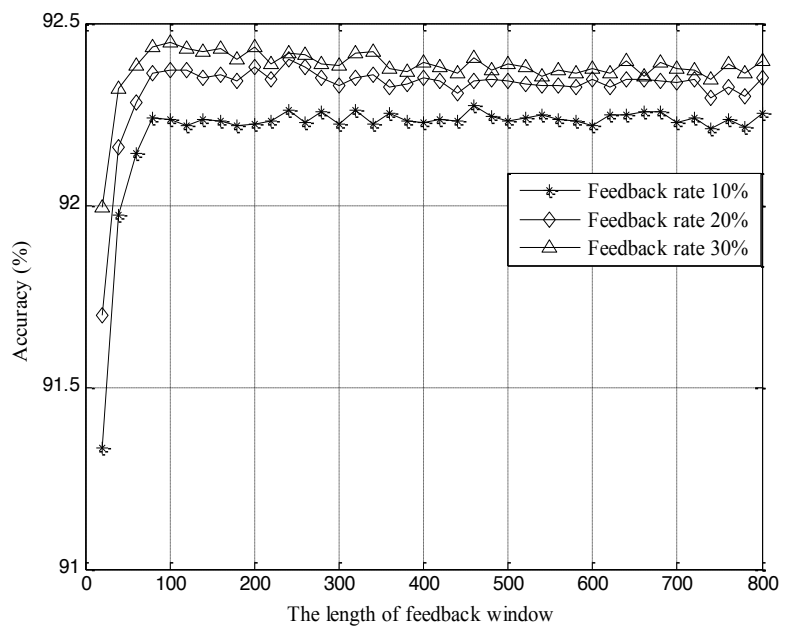

Fig. (2e). The accuracy versus the length of feedback window for the $5^{\text {th }}$ group of data.

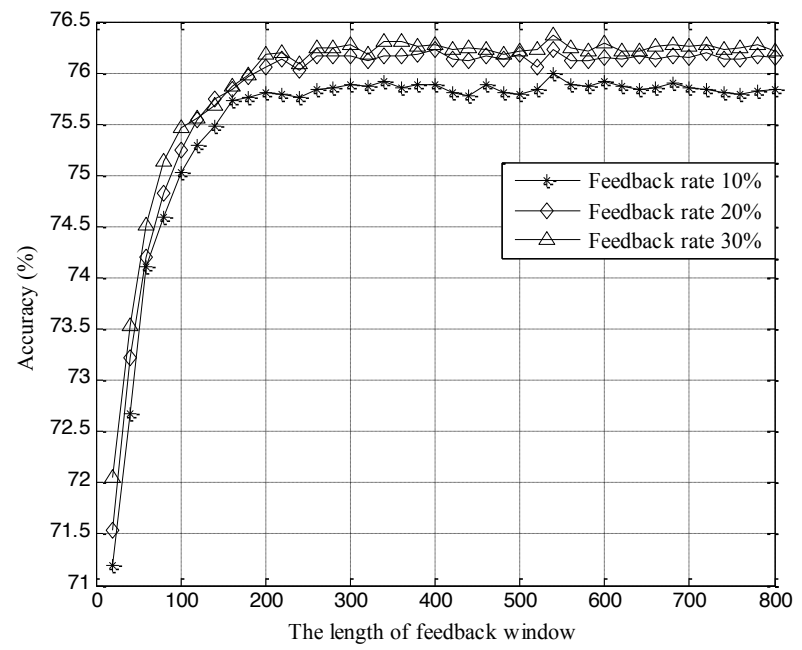

Fig. (2f). The accuracy versus the length of feedback window for the $6^{\text {th }}$ group of data. 
Table 2. The error conditions of sensors' data in simulation of the length of feedback window.

\begin{tabular}{|c|c|c|c|}
\hline & Sensor No. & Error Rate(\%) & Burst Error Length \\
\hline \multirow{3}{*}{ The First Group } & Sensor 1 & 15 & - \\
\hline & Sensor 3 & 15 & - \\
\hline & Sensor 4 & 15 & 300 \\
\hline \multirow[b]{3}{*}{ The Second Group } & Sensor 1 & 15 & - \\
\hline & Sensor 2 & 25 & - \\
\hline & Sensor 3 & 35 & - \\
\hline \multirow{5}{*}{ The Third Group } & Sensor 1 & 35 & - \\
\hline & Sensor 2 & 35 & - \\
\hline & Sensor 3 & 35 & - \\
\hline & Sensor 4 & 35 & 300 \\
\hline & Sensor 5 & 35 & 300 \\
\hline \multirow{2}{*}{ The Fourth Group } & Sensor 1 & 15 & - \\
\hline & Sensor 2 & 15 & - \\
\hline \multirow[t]{3}{*}{ The Fifth Group } & Sensor 3 & 35 & - \\
\hline & Sensor 4 & 25 & - \\
\hline & Sensor 5 & 15 & - \\
\hline \multirow{5}{*}{ The Sixth Group } & Sensor 1 & 35 & - \\
\hline & Sensor 2 & 35 & - \\
\hline & Sensor 3 & 35 & - \\
\hline & Sensor 4 & 35 & - \\
\hline & Sensor 5 & 35 & - \\
\hline
\end{tabular}

From Fig. (2d) and Fig. (2e) it can be seen that when the length of window is less than 100, the accuracy of the proposed algorithm improves sharply as the window length increases, and when the length of window exceeds 100 , the accuracy becomes stabilized.
From Fig. (2f) it can be seen that when the length of window is less than 300, the accuracy of the proposed algorithm improves sharply as the window length increases, and when the length of window exceeds 300, the accuracy becomes stabilized. 
Table 3. The error conditions of sensors' data in simulation of accuracy performance.

\begin{tabular}{|c|c|c|c|}
\hline & Sensor No. & Error Rate(\%) & Burst Error Length \\
\hline \multirow{5}{*}{ The First Group } & Sensor 1 & $1 \sim 40$ & - \\
\hline & Sensor 2 & 15 & - \\
\hline & Sensor 3 & 15 & - \\
\hline & Sensor 4 & 15 & 300 \\
\hline & Sensor 5 & 15 & 300 \\
\hline \multirow{5}{*}{ The Second Group } & Sensor 1 & $1 \sim 40$ & - \\
\hline & Sensor 2 & 25 & - \\
\hline & Sensor 3 & 35 & - \\
\hline & Sensor 4 & 25 & 300 \\
\hline & Sensor 5 & 15 & 300 \\
\hline \multirow{5}{*}{ The Third Group } & Sensor 1 & $1 \sim 40$ & - \\
\hline & Sensor 2 & 35 & - \\
\hline & Sensor 3 & 35 & - \\
\hline & Sensor 4 & 35 & 300 \\
\hline & Sensor 5 & 35 & 300 \\
\hline \multirow{5}{*}{ The Fourth Group } & Sensor 1 & $1 \sim 40$ & - \\
\hline & Sensor 2 & 15 & - \\
\hline & Sensor 3 & 15 & - \\
\hline & Sensor 4 & 15 & - \\
\hline & Sensor 5 & 15 & - \\
\hline \multirow{5}{*}{ The Fifth Group } & Sensor 1 & $1 \sim 40$ & - \\
\hline & Sensor 2 & 25 & - \\
\hline & Sensor 3 & 35 & - \\
\hline & Sensor 4 & 25 & - \\
\hline & Sensor 5 & 15 & - \\
\hline \multirow{5}{*}{ The Sixth Group } & Sensor 1 & $1 \sim 40$ & - \\
\hline & Sensor 2 & 35 & - \\
\hline & Sensor 3 & 35 & - \\
\hline & Sensor 4 & 35 & - \\
\hline & Sensor 5 & 35 & - \\
\hline
\end{tabular}

Through the comprehensive analysis of Fig. (2d-f), it can be seen that if the data only contain random errors, the accuracy of the proposed algorithm is relatively higher when the length of feedback window is bigger than 50, and the algorithm's accuracy would increase in a specific range of short window length when the length of window becomes larger, which is especially right for the higher random error rate (like 6th Group). Moreover, when the length of window exceeds a certain value, the accuracy becomes stabilized because the feedback error rate can truly reflect the condition of the random errors.

By analyzing the above simulation results about the relationship between the length of feedback window and the accuracy of the proposed algorithm comprehensively, and considering different kinds of error conditions, i.e., with both random errors and burst errors or with only random errors, we finally choose the suitable length of feedback window as 


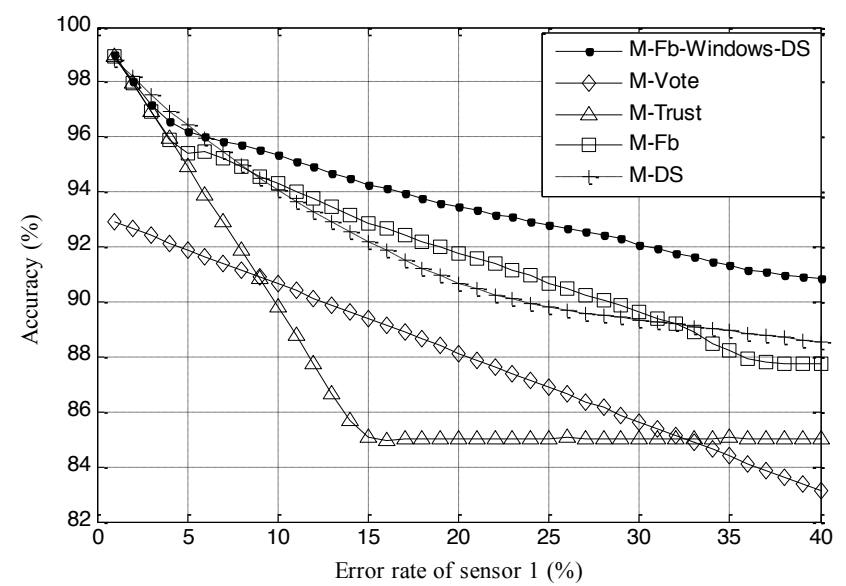

Fig. (3a). The accuracy of five algorithms versus the error rate of sensor 1 in the $1^{\text {st }}$ data group.

75. In addition, we may also choose the feedback rate as $20 \%$ because the system can get relative good accuracy but low feedback cost at this rate.

In the following simulations, we will compare the performance of the proposed algorithm based on feedback windowing and DS evidence theory (M-Fb-Windows-DS) with other four context inconsistency elimination algorithms, including the voting based algorithm (M-Vote) [8], the trustworthiness based algorithm (M-Trust) [9], the D-S evidence theory based algorithm (M-DS) [10], and the user feedback based algorithm (M-Fb) [11].

We evaluate the performance of the context inconsistency elimination algorithms based on different kinds of errors. Here we assume that the system acquires six groups of data in which three groups contain burst errors while the others do not, and the error conditions of sensors' data are shown in Table 3. Notice that, the data in Table $\mathbf{3}$ are similar to that of Table 2, but the error conditions of sensor 1 in six groups of data are set to be $1 \sim 40 \%$ other than the fixed value, so we can evaluate the accuracy of the five algorithms versus the error rate of sensor 1 in different data groups. Of course, we can use other sensor's data to do the analysis, which would be similar, so here we make the sensor 1 as an example to do the following simulations.

Consider the actual application occasions of smart home and the above simulation results of some key parameters, we assume that $T h_{a c c}$ and $T h_{r e j}$ are 5\% and 50\% respectively, and set the feedback rate and the length of feedback window to be $20 \%$ and 75 , respectively.

As shown in Fig. (3a), for the five different algorithms, the accuracies of M-Vote algorithm and M-Trust algorithm are relatively lower compared with other three algorithms, and the accuracies of $\mathrm{M}-\mathrm{Fb}$ algorithm and $\mathrm{M}-\mathrm{DS}$ algorithm are similar. For the proposed M-Fb-Windows-DS algorithm, when the data error rate of sensor 1 is between $1 \%$ and $7 \%$, it has the similar accuracy with the M-DS algorithm which is the best one among the other four typical algorithms; and when the data error rate of sensor 1 exceeds $7 \%$, its accuracy begins to exceed all the other four algorithms. For example, when the data error rate of sensor 1 equals $32 \%$, the accuracy of the proposed algorithm rises $2.6 \%$ compared with the best one obtained by the other four algorithms, i.e. $\mathrm{M}-\mathrm{Fb}$ algorithm.

As shown in Fig. (3b), for the five different algorithms, the performance of M-Vote algorithm is the worst, and the other three typical algorithms used for comparison have similar accuracies. For the proposed $\mathrm{M}-\mathrm{Fb}$-Windows-DS algorithm, when the data error rate of sensor 1 is between $1 \%$ and $9 \%$, it has almost the same accuracy as the M-Trust algorithm which is the best one among the other four typical algorithms; and when the data error rate of sensor 1 exceeds $9 \%$, its accuracy begins to exceed all the other four algorithms. For example, when the data error rate of sensor 1 equals $29 \%$, the accuracy of the proposed algorithm rises $4.68 \%$ compared with the best one obtained by the other four algorithms, i.e. $\mathrm{M}-\mathrm{Fb}$ algorithm.

As shown in Fig. (3c), the performance of M-Vote algorithm is the worst, and the other three typical algorithms used for comparison have similar accuracies. For the proposed $\mathrm{M}-\mathrm{Fb}$-Windows-DS algorithm, when the data error rate of sensor 1 is between $1 \%$ and $23 \%$, it has almost the same accuracy as the M-Trust algorithm which is the best one among the other four typical algorithms; and when the data error rate of sensor 1 exceeds $23 \%$, its accuracy begins to exceed all the other four algorithms. For example, when the data error rate equals $36 \%$, the accuracy of the $\mathrm{M}-\mathrm{Fb}$ Windows-DS algorithm rises $2.24 \%$ compared with the best one obtained by the other algorithms, i.e. M-Fb algorithm.

From Fig. (3a-c) we can see that, when context information contains burst errors, the accuracy of the proposed $\mathrm{M}-\mathrm{Fb}$-Windows-DS algorithm is higher than other four typical algorithms. The reason is that, through the using of window mechanism, the proposed algorithm can reflect the burst errors timely and enhance the ability to resist burst errors; meanwhile, by using the evidence theory, the proposed algorithm can further improve the accuracy of context inconsistency elimination. 


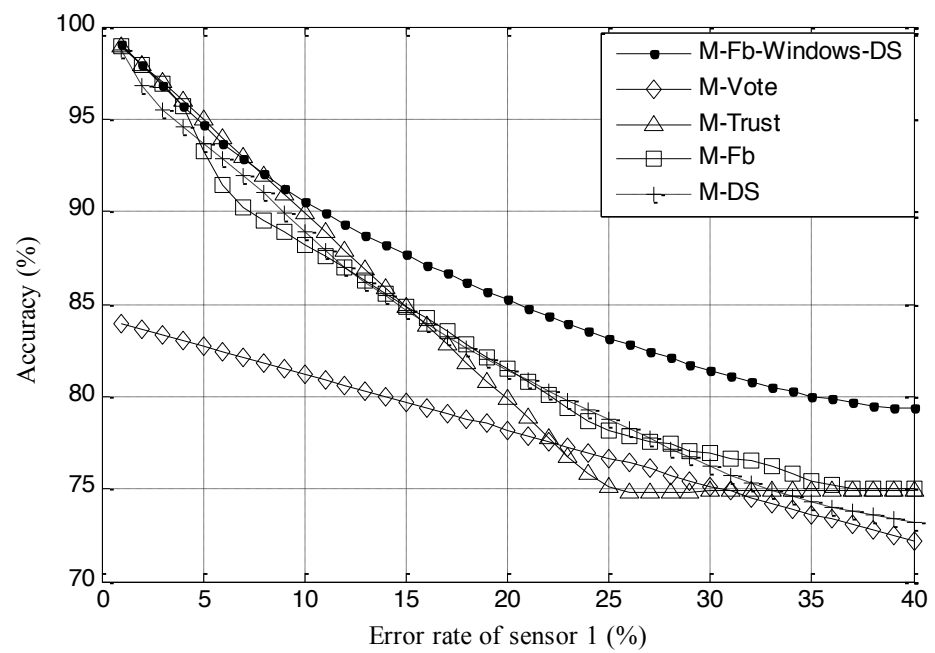

Fig. (3b). The accuracy of five algorithms versus the error rate of sensor 1 in the $2^{\text {nd }}$ data group.

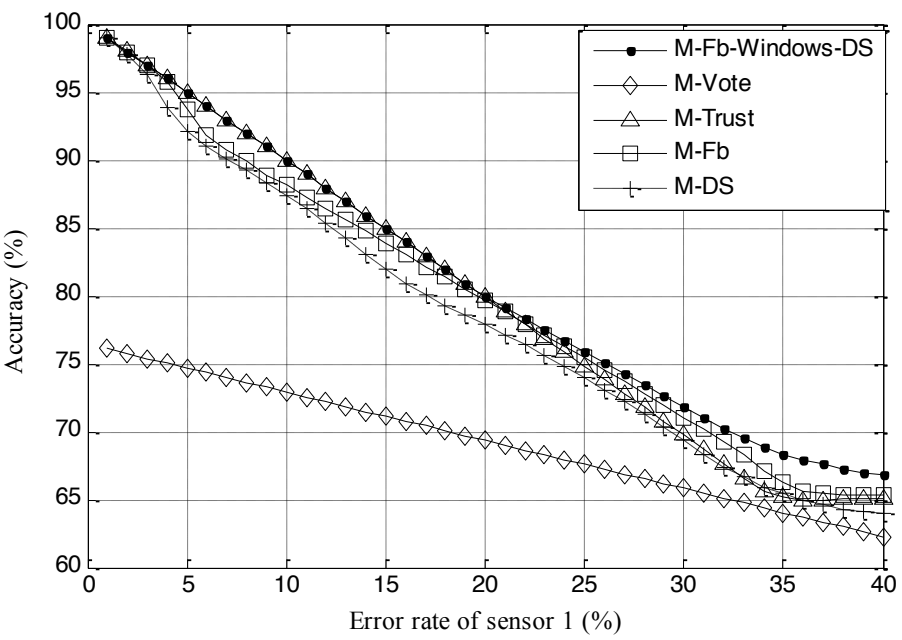

Fig. (3c). The accuracy of five algorithms versus the error rate of sensor 1 in the $3^{\text {rd }}$ data group.

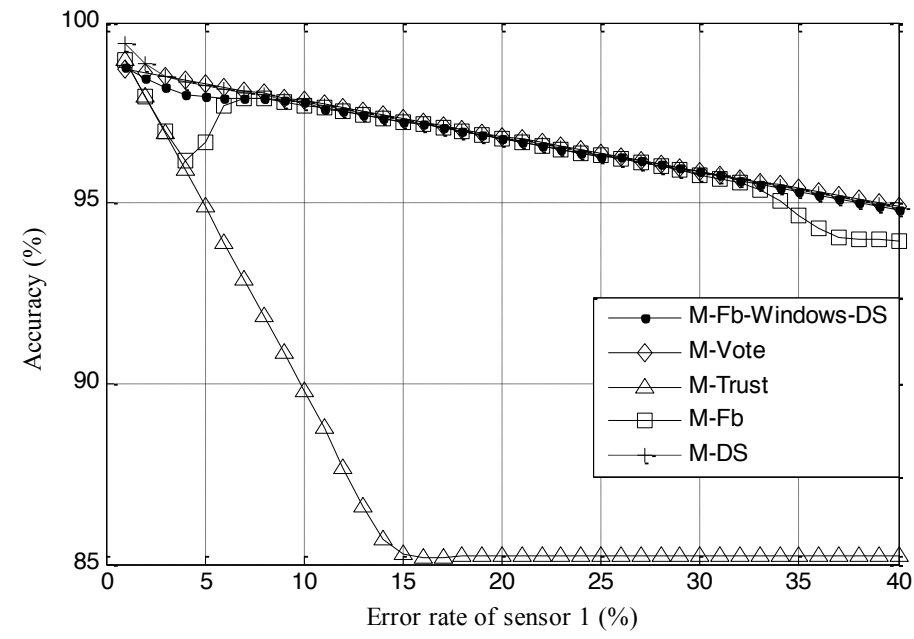

Fig. (3d). The accuracy of five algorithms versus the error rate of sensor 1 in the $4^{\text {th }}$ data group. 


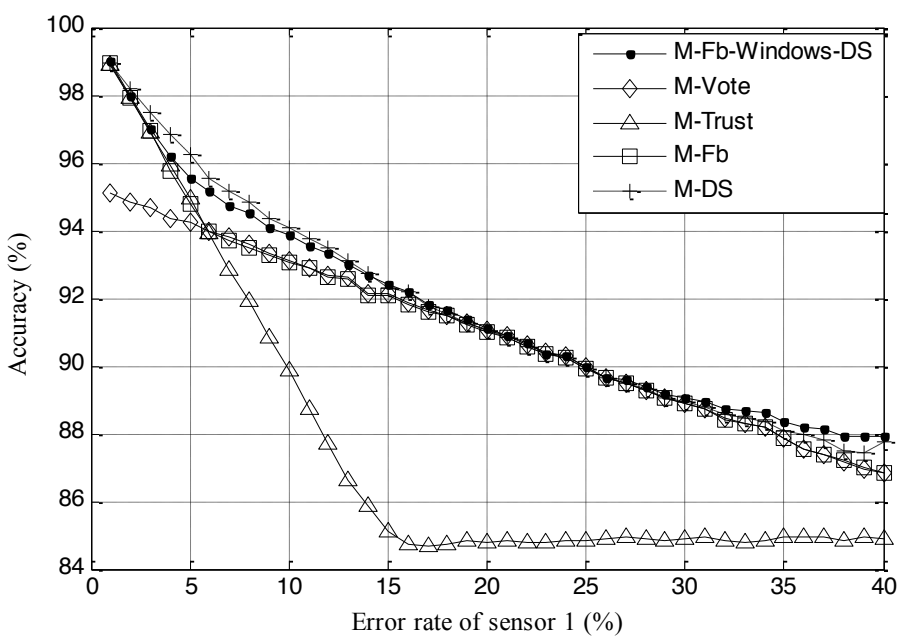

Fig. (3e). The accuracy of five algorithms versus the error rate of sensor 1 in the $5^{\text {th }}$ data group.

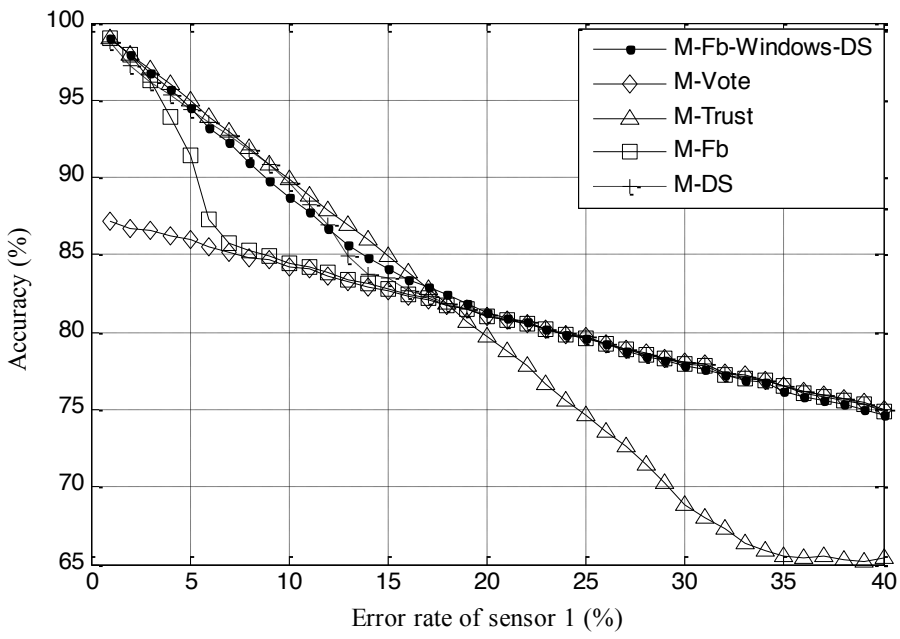

Fig. (3f). The accuracy of five algorithms versus the error rate of sensor 1 in the $6^{\text {th }}$ data group.

As shown in Fig. (3d), for the five different algorithms, the performance of M-Trust algorithm is the worst, and the other typical three algorithms used for comparison have similar accuracies. For the proposed M-Fb-Windows-DS algorithm, when the data error rate of sensor 1 is between $1 \%$ and $10 \%$, it has almost the same accuracy as M-Trust and M$\mathrm{Fb}$ algorithms, which is higher than that of M-Trust and M$\mathrm{Fb}$ algorithms; and when the data error rate of sensor 1 exceeds $10 \%$, it has similar performance with $\mathrm{M}-$ Vote, $\mathrm{M}-\mathrm{Fb}$ and M-DS algorithms, and its performance is better than that of M-Trust algorithm.

As shown in Fig. (3e), for the five different algorithms, the performance of M-Trust algorithm is the worst. For the proposed $\mathrm{M}-\mathrm{Fb}$-Windows-DS algorithm, when the data error rate of sensor 1 is between $1 \%$ and $14 \%$, it has almost the same accuracy as M-DS algorithm which is the best one among the other four typical algorithms; and when the data error rate of sensor 1 is between $14 \%$ and $30 \%$, it has similar performance with M-Vote, M-Fb and M-DS algorithms, which is better than M-Trust algorithm; and when the data error rate of sensor 1 exceeds $30 \%$, its accuracy begins to exceed all the other four algorithms.

As shown in Fig. (3f), for the proposed M-Fb-WindowsDS algorithm, when the data error rate of sensor 1 is between $1 \%$ and $17 \%$, it has almost the same accuracy as the M-Trust and M-DS algorithms, which is higher than that of M-Vote and $\mathrm{M}-\mathrm{Fb}$ algorithms; and when the data error rate of sensor 1 exceeds $17 \%$, it has similar performance with M-Vote, M$\mathrm{Fb}$ and M-DS algorithms, its performance is better than MTrust algorithm.

By analyzing the above simulation results about the accuracy of several algorithms under different data error rates, we can know that, in most cases, the performance of accuracy of the proposed $\mathrm{M}-\mathrm{Fb}$-Windows-DS algorithm is better than the other four kinds of typical algorithms, especially when the context data contain the burst errors. The proposed algorithm improves the ability of context-aware system to deal with burst errors by executing the windowing processing, and it gets obvious improvement on context inconsistency elimination. 


\section{CONCLUSION}

This paper presents a new context inconsistency elimination algorithm based on feedback windowing and evidence theory, and also analyzes the optimal values of some parameters of the proposed algorithm on the basis of simulations. The performance of the proposed algorithm is evaluated under the conditions of different data error rates and compared with four kinds of typical algorithms. The simulation results show that the accuracy of the proposed algorithm is superior to the other four typical algorithms when context information contains burst errors. What's more, the overall performance of accuracy of the proposed algorithm can increase at least $2 \% \sim 5 \%$ for different error rates of context information.

\section{CONFLICT OF INTEREST}

The authors confirm that this article content has no conflict of interest.

\section{ACKNOWLEDGEMENTS}

The research work was supported by Scientific Research Foundation for the Excellent Young and Middle-aged Scientists of Shandong Province (BS2012DX024), Independent Innovation Foundation of Shandong University (2012ZD035), Technical Innovative Project of Shandong Province (201230201031, 201320201024).
[2] P. Makris, D. N. Skoutas, and C. Skianis, "A survey on contextaware mobile and wireless networking:on networking and computing environments' integration", Communications Surveys and Tutorials, 15 , no. 1 , pp. $362-86,2013$

[3] J. Park, and K.H. Lee, "Reaping context-aware services from living data" Proceedings of International Conference on Information Science and Applications: Waltham 1-3, 2013

[4] Y. Nam, S. Rho, and B. Lee, "Intelligent context-aware energy management using the incremental simultaneous method in future wireless sensor networks and computing systems", EURASIP Journal on Wireless Communications and Networking, vol. 10, pp. 111,2013

[5] B. Hu, Z. Wang, Q. Dong, and Y. Niu, "Context-aware knowledge acquisition and reasoning based on description logic", Computer Science, vol. 40, no. 4, pp. 199-203, 2013

[6] X. Chen, C. Xu, and L. Jiang, "Automated fixing of inconsistent contexts" Journal of Frontiers of Computer Science and Technology, vol. 7, no. 4, pp. 326-36, 2013.

[7] C. Chen, C. Ye, and H. A. Jacobsen, "Hybrid context inconsistency resolution for context-aware services", Proceedings of IEEE International Conference on Pervasive Computing and Communications: Seattle pp. 10-9, 2011

[8] D. Zheng, and S. Zhu, "Research of algorithms for elimination of inconsistent context in pervasive computing", Application Research of Computes, vol. 26, no. 1, pp. 152-4, 2009.

[9] A. Manzoor, H. L. Truong, and S. Dustdar, On the Evaluation of Quality of Context Smart Sensing and Context (Lecture Notes in Computer Science), Springer: Berlin Heidelberg, 2008, pp. 140-53,

[10] L. Jousselme, D. Grenier, and E. Bossé, "A new distance between two bodies of evidence", Information Fusion, vol. 2, no. 2, pp. 91$101,2001$.

[11] H. Lee, and D. H. Kim, "Efficient context-aware selection based on user feedback", IEEE Transactions on Consumer Electronics, vol. 58, no. 3, pp. 978-84, 2012

[12] G. Shafer, A Mathematical Theory of Evidence, Princeton University Press: Princeton, NJ, 1976.

\section{REFERENCES}

[1] H. Viswanathan, B. Chen, and D. Pompili, "Research challenges in computation, communication, and context awareness for ubiquitous healthcare", Communications Magazine, vol. 50, no. 5, pp. 92-9, 2012.

Received: November 21, 2014

Revised: January 07, 2015

Accepted: January 20, 2015

(C) Xu et al.; Licensee Bentham Open.

This is an open access article licensed under the terms of the Creative Commons Attribution Non-Commercial License (http://creativecommons.org/licenses/by$\mathrm{nc} / 3.0 /$ ) which permits unrestricted, non-commercial use, distribution and reproduction in any medium, provided the work is properly cited. 